\title{
Chronic obstructive pulmonary disease and coronary artery bypass grafting
}

\section{La maladie pulmonaire obstructive chronique et le pontage aortocoronarien}

I

$\mathrm{n}$ the present issue of the Canadian Respiratory Journal, Manganas et al (1) published a study of coronary artery bypass grafting (CABG) in patients with and without chronic obstructive pulmonary disease (COPD). This is an important subject, because coronary artery disease and COPD are both related to smoking and therefore frequently coexist; many COPD patients die of coronary artery disease (2). In any event, Manganas et al identified more than 300 patients who had spirometry soon before undergoing CABG and categorized them into three groups: those with normal spirometry results; those with mild-moderate COPD (forced expiratory volume in $1 \mathrm{~s}\left[\mathrm{FEV}_{1}\right] /$ forced vital capacity [FVC] 0.7 or less, $\mathrm{FEV}_{1}$ of at least $50 \%$ of the predicted normal value); and severe COPD $\left(\mathrm{FEV}_{1} / \mathrm{FVC} 0.7\right.$ or less, $\mathrm{FEV}_{1}$ less than $50 \%$ of the predicted value). The mean $\mathrm{FEV}_{1}$ in the last group was $0.98 \mathrm{~L}$. The groups were well balanced in terms of cardiac disease severity and use of cardiac drugs, and had similar surgery, although internal mammary artery grafts were used less often in both COPD groups. Mortality was very low in all groups and not related to COPD; indeed, none of the 68 severe COPD patients died. None of the seven deaths were due to respiratory disease. On the other hand, COPD patients had slightly longer hospital stays than those with normal spirometry, and had more episodes of bronchitis and pneumonia - approximately $25 \%$ of the severe COPD patients had one of these complications. As Manganas et al indicate, these are fairly soft diagnoses in such a retrospective study. Furthermore, there was a tendency for atrial fibrillation to occur more often in COPD patients.

Consultations with respirologists increased with the severity of the preoperative COPD, but did not influence the postoperative result, a sobering finding.

These results contrast with older studies (cited by Manganas et al) in several ways. First, CABG mortality has decreased strikingly over the past 15 to 20 years, and second, a preoperative diagnosis of COPD did not appear to impose serious added risks to CABG. Indeed, Manganas et al did not find significant added risk when they looked at patients with $\mathrm{FEV}_{1}$ values less than $35 \%$ of predicted. The preoperative diagnosis may have influenced the behaviour of the surgical team in a way that minimized complications. An example of this is the (slightly) reduced frequency of internal mammary artery grafts, known to be associated with postoperative pulmonary problems (3). On the other hand, it is equally possible that the preoperative diagnosis sensitized the surgical team to the possibility of postoperative bronchitis and pneumonia, and that the true incidence of these problems was actually lower than that reported. It is not clear whether these results apply to patients with undiagnosed COPD, ie, those who have not had preoperative spirometry, ans le présent numéro du Canadian Respiratory Uournal, Manganas et coll. (1) publient une étude sur le pontage aortocoronarien (PAC) chez les patients atteints ou non de maladie pulmonaire obstructive chronique (MPOC). C'est un sujet important, parce que les coronaropathies et les MPOC sont toutes deux reliées au tabagisme et coexistent donc souvent. De nombreuses personnes atteintes d'une MPOC meurent d'une coronaropathie. (2) Quoi qu'il en soit, Manganas et coll. ont repéré plus de 300 patients qui avaient subi une spirométrie peu avant de subir une PAC et les ont divisés en trois groupes : ceux qui avaient obtenu des résultats normaux à la spirométrie, ceux qui étaient atteints d'une MPOC bénigne à moyenne (volume expiratoire maximal par seconde [VEMS]/capacité vitale forcée [CVF] de 0,7 ou moins, VEMS d'au moins $50 \%$ de la valeur normale prévue), et ceux qui étaient atteints d'une MPOC grave moyenne (VEMS/CVF de 0,7 ou moins, VEMS de moins de $50 \%$ de la valeur normale prévue). Le VEMS moyen au sein du dernier groupe était de 0,98 L. Les groupes étaient bien équilibrés pour ce qui est de la gravité de la maladie cardiaque et de l'utilisation de médicaments cardiaques, et ils avaient subi des opérations similaires, bien que les pontages de l'artère mammaire interne aient été moins fréquents dans les deux groupes de MPOC. La mortalité était très faible dans les deux groupes et n'était pas reliée à la MPOC. En fait, aucun de 68 patients atteints d'une MPOC grave n'est décédé. Aucun des sept décès n'était imputable à une maladie respiratoire. Par contre, les patients atteints d'une MPOC étaient hospitalisés légèrement plus longtemps que ceux dont la spirométrie était normale, et ils avaient plus d'épisodes de bronchite et de pneumonie, car environ $25 \%$ de ces patients souffraient de l'une de ces complications. Comme l'ont indiqué Manganas et coll., ce sont des diagnostics plutôt cléments dans une telle étude rétrospective. De plus, on remarquait une plus forte tendance à la fibrillation auriculaire chez les patients atteints d'une MPOC.

Les consultations de pneumologues augmentaient avec la gravité de la MPOC préopératoire mais n'influaient pas sur le résultat postopératoire, ce qui est une constatation qui fait réfléchir.

Ces résultats contrastent avec ceux d'études plus anciennes (citées par Manganas et coll.) sous divers aspects. En premier lieu, la mortalité secondaire aux PAC a considérablement diminué depuis 15 à 20 ans, et en deuxième lieu, un diagnostic préopératoire de MPOC ne semblait pas s'associer à des risques supplémentaires graves en cas de PAC. De fait, Manganas et coll. n'ont constaté aucun risque supplémentaire significatif lorsqu'ils ont observé des patients dont la VEMS était de $35 \%$ inférieure à celle prévue. Le diagnostic préopératoire peut avoir influé sur le comportement de l'équipe chirurgicale de manière 
and one fears that this may be common. Such patients may do less well, but this is by no means certain.

Of particular interest to me is the increased incidence of atrial fibrillation in the COPD patients. In database studies, we have found that this arrhythmia was associated with the use of short-acting bronchodilators (4) in COPD patients. Whether this is due to the drugs or to the associated disease is difficult to say, but it is worth thinking about. Manganas et al did not find such a relationship among their patients.

Nick R Anthonisen MD Editor-in-Chief, Canadian Respiratory Journal

\section{REFERENCES}

1. Manganas H, Lacasse Y, Bourgeois S, Perron J, Dagenais F, Maltais F. Postoperative outcome after coronary artery bypass grafting in chronic obstructive pulmonary disease. Can Respir J 2007;14:19-24.

2. Sin DD, Anthonisen NR, Soriano JB, Agusti AG. Mortality in COPD: Role of comorbidities. Eur Respir J 2006;28:1245-57.

3. Ng CS, Wan S, Yim AP, Arifi AA. Pulmonary dysfunction after cardiac surgery. Chest 2002;121:1269-77.

4. Macie C, Wooldrage K, Manfreda J, Anthonisen NR. Risk of cardiovascular events associated with use of inhaled ipratropium in respiratory disease. Proc Am Thoracic Soc 2004;1:A520. (Abst) à réduire les complications au minimum. La fréquence (légèrement) réduite de pontages de l'artère mammaire interne est un exemple de ce constat, car on sait ce pontage associé à des troubles pulmonaires postopératoires (3). Par contre, il est tout aussi possible que le diagnostic préopératoire ait sensibilité l'équipe chirurgicale à la possibilité de bronchite et de pneumonie postopératoire et que la véritable incidence de ces troubles ait été moins élevée que celle qui a été déclarée. On ne sait pas si ces résultats s'appliquent aux patients atteints d'une MPOC non diagnostiquée, c'est-à-dire ceux qui n'ont pas subi de spirométrie avant l'opération. Il est à craindre que ces troubles soient alors courants. Ces patients s'en tirent peut-être moins bien, mais ce n'est pas du tout certain.

Je trouve particulièrement intéressante l'incidence accrue de fibrillation auriculaire chez les patients atteints de MPOC. Dans les études des bases de données, on a découvert que cette arythmie s'associait au recours de bronchodilatateurs à court terme (4) chez les patients atteints de MPOC. Il est difficile de savoir si ce phénomène est causé par les médicaments ou par la maladie connexe, mais ça mérite réflexion. Manganas et coll. n'ont pas constaté de telle relation chez leurs patients.

Nick R. Anthonisen MD Rédacteur en chef, Canadian Respiratory Journal

\section{RÉFÉRENCES}

1. Manganas H, Lacasse Y, Bourgeois S, Perron J, Dagenais F, Maltais F. Postoperative outcome after coronary artery bypass grafting in chronic obstructive pulmonary disease. Can Respir J 2007;14:19-24.

2. Sin DD, Anthonisen NR, Soriano JB, Agusti AG. Mortality in COPD: Role of comorbidities. Eur Respir J 2006;28:1245-57.

3. Ng CS, Wan S, Yim AP, Arifi AA. Pulmonary dysfunction after cardiac surgery. Chest 2002;121:1269-77.

4. Macie C, Wooldrage K, Manfreda J, Anthonisen NR. Risk of cardiovascular events associated with use of inhaled ipratropium in respiratory disease. Proc Am Thoracic Soc 2004;1:A520. (Abst) 


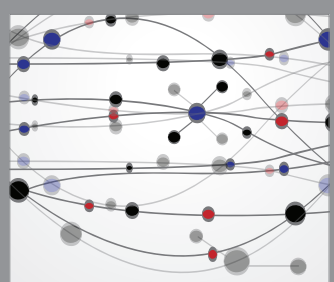

The Scientific World Journal
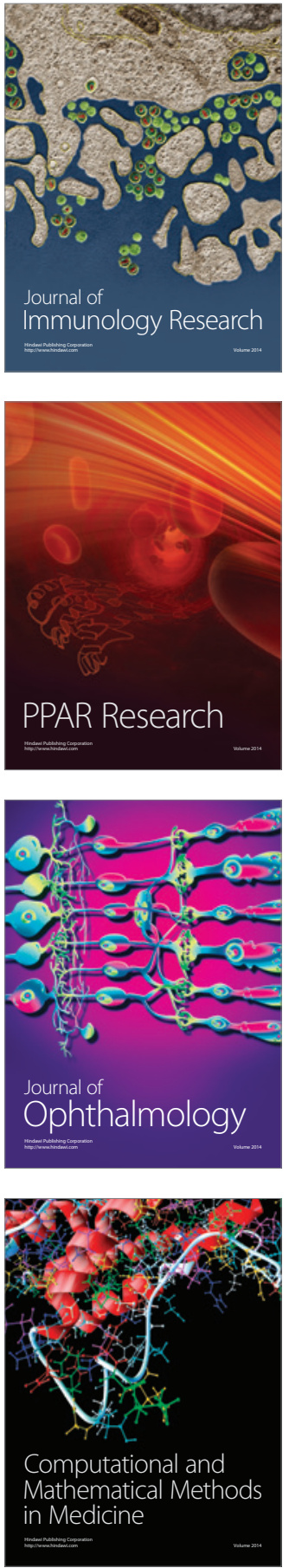

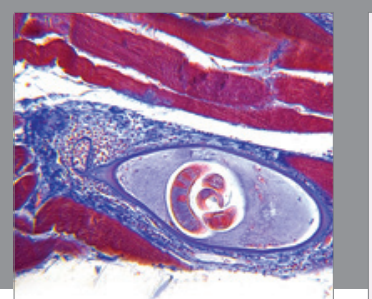

Gastroenterology Research and Practice

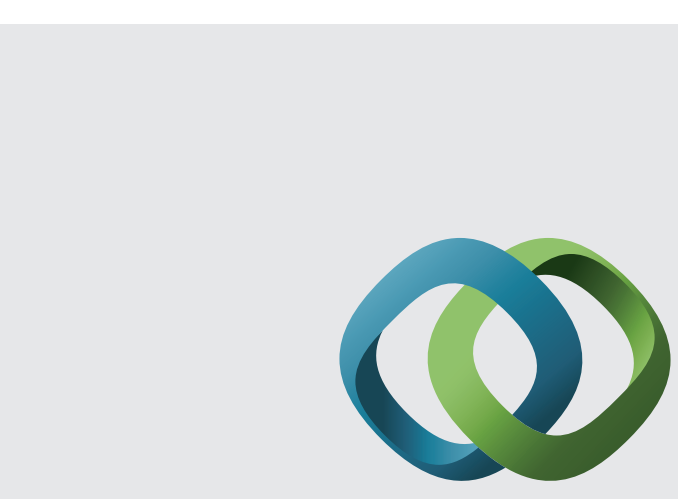

\section{Hindawi}

Submit your manuscripts at

http://www.hindawi.com
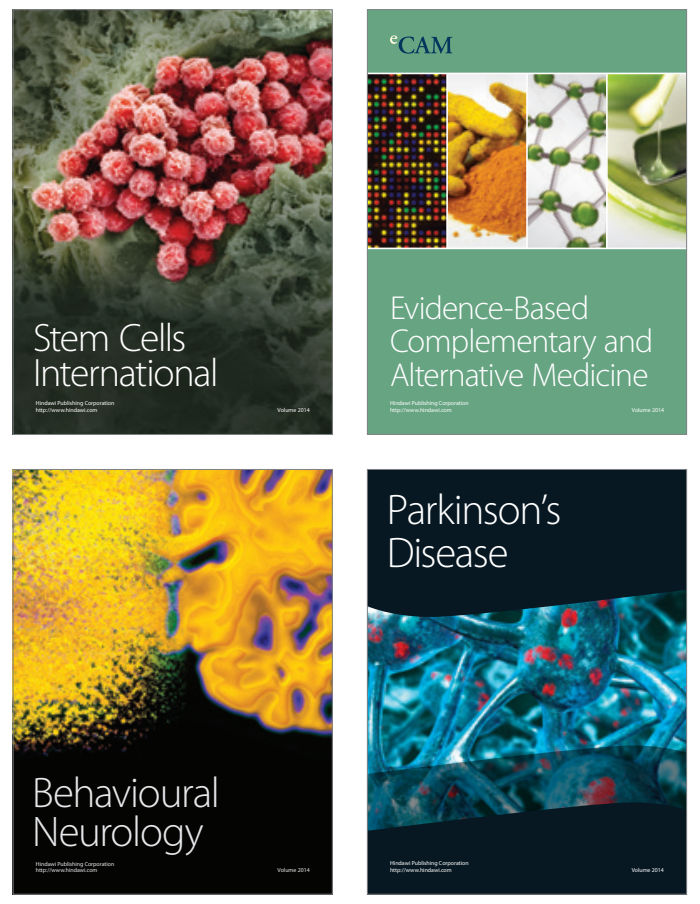
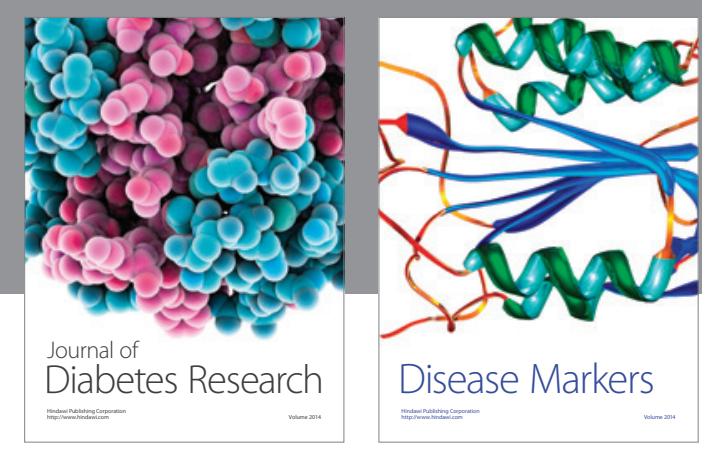

Disease Markers
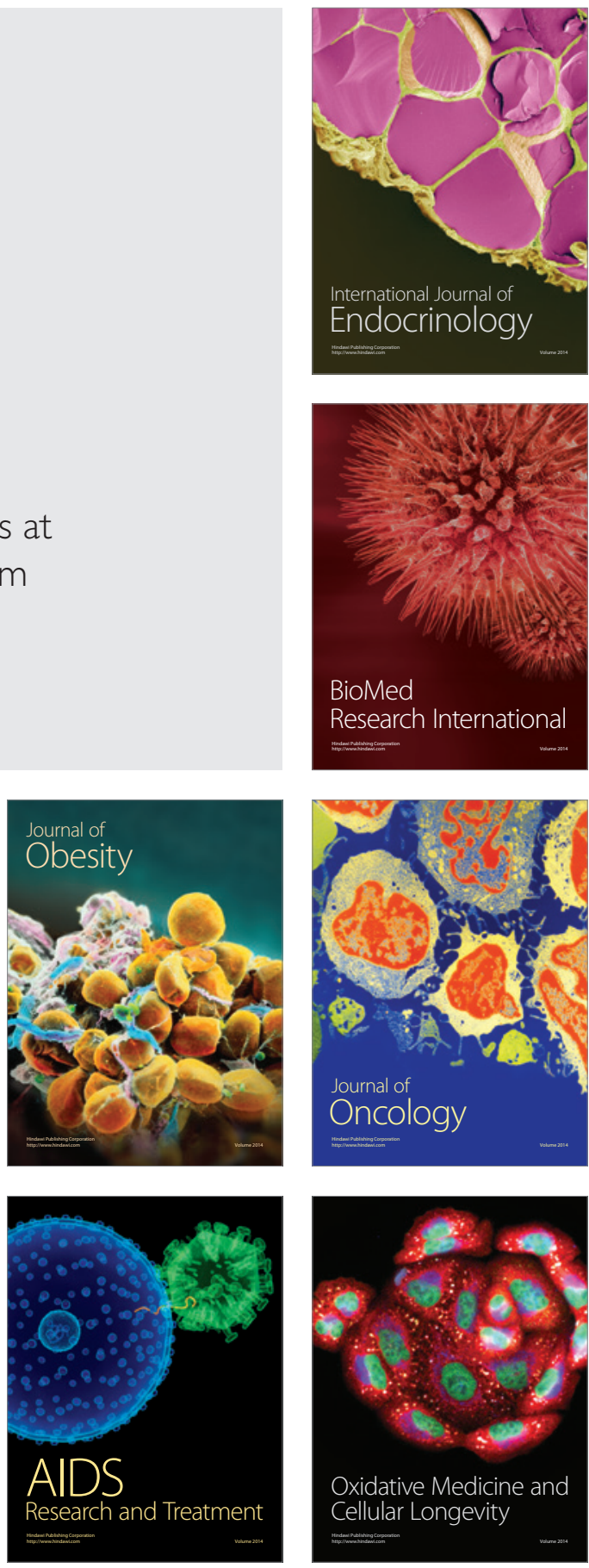